\title{
Impaired Learning From Errors in Cannabis Users: Dorsal Anterior Cingulate Cortex and Hippocampus Hypoactivity*
}

\author{
Susan E Carey ${ }^{\mathrm{a}}$, Liam Nestor ${ }^{\mathrm{b}}$, Jennifer Jones $^{\mathrm{b}}$, Hugh Garavan ${ }^{\mathrm{b}, \mathrm{c}}$, and Robert Hester ${ }^{\mathrm{a}}$ \\ aSchool of Psychological Sciences, University of Melbourne, Melbourne, Victoria, Australia \\ bSchool of Psychology and Trinity College Institute of Neuroscience, Trinity College Dublin, \\ Dublin, Ireland 'Departments of Psychiatry and Psychology, University of Vermont, USA
}

\begin{abstract}
Background-The chronic use of cannabis has been associated with error processing dysfunction, in particular, hypoactivity in the dorsal anterior cingulate cortex (dACC) during the processing of cognitive errors. Given the role of such activity in influencing post-error adaptive behaviour, we hypothesised that chronic cannabis users would have significantly poorer learning from errors.
\end{abstract}

Methods-Fifteen chronic cannabis users (four females, mean age $=22.40$ years, $S D=4.29$ ) and 15 control participants (two females, mean age $=23.27$ years, $S D=3.67$ ) were administered a paired associate learning task that enabled participants to learn from their errors, during fMRI data collection.

Results-Compared with controls, chronic cannabis users showed (i) a lower recall errorcorrection rate and (ii) hypoactivity in the $\mathrm{AACC}$ and left hippocampus during the processing of error-related feedback and re-encoding of the correct response. The difference in error-related dACC activation between cannabis users and healthy controls varied as a function of error type, with the control group showing a significantly greater difference between corrected and repeated errors than the cannabis group.

Conclusions-The present results suggest that chronic cannabis users have poorer learning from errors, with the failure to adapt performance associated with hypoactivity in error-related dACC and hippocampal regions. The findings highlight a consequence of performance monitoring dysfunction in drug abuse and the potential consequence this cognitive impairment has for the

\footnotetext{
* Supplementary material can be found by accessing the online version of this paper at http://dx.doi.org and by entering doi:... Address correspondence to: Robert Hester, Melbourne School of Psychological Sciences, Redmond Barry Building, University of Melbourne, Parkville, Victoria, 3010, Australia. hesterr@unimelb.edu.au Phone: +61 (0)3 8344 6377. Fax: +61 393476618.

Contributors RH and HG designed the study and wrote the protocol, RH and SC analysed data and wrote the first draft of the manuscript; LN and JJ collected the behavioural and fMRI data, and assisted with design and interpretation of the data. All authors contributed to and have approved the final manuscript.

Conflicts of Interest: Ms. Carey, Drs. Nestor, Jones, Garavan, and Hester reported no biomedical financial interests or potential conflicts of interest.

Publisher's Disclaimer: This is a PDF file of an unedited manuscript that has been accepted for publication. As a service to our customers we are providing this early version of the manuscript. The manuscript will undergo copyediting, typesetting, and review of the resulting proof before it is published in its final citable form. Please note that during the production process errors may be discovered which could affect the content, and all legal disclaimers that apply to the journal pertain.
} 
symptom of failing to learn from negative feedback seen in cannabis and other forms of dependence.

\section{Keywords}

Addiction; cannabis; dorsal anterior cingulate cortex; drug addiction; fMRI

\section{INTRODUCTION}

The chronic use of cannabis has been associated with a range of cognitive impairments, including impairments in learning, memory, and executive functions (Crean et al., 2011; Ranganathan and D'Souza, 2006), with a dose-response relationship between chronicity of cannabis consumption and deterioration in these cognitive domains (Messinis et al., 2006). While there remains equivocation regarding the specific nature of the executive function impairments in the cannabis-using population (Grant et al., 2002) - likely due to diverse methodologies and measures employed (Verdejo-García et al., 2004)— there is more consistent evidence of impairments in learning (see Solowij and Battisti, 2008 for a review). Of particular interest to the current study is dysfunction in error learning in chronic cannabis users (CCU), because difficulty in adjusting behaviour in the face of negative consequences is a core clinical symptom of cannabis dependence and other drug dependencies (Kalivas and Volkow, 2005).

Error processing refers to monitoring performance, detecting errors, and modifying behaviour adaptively in the absence of overt reinforcement (Holroyd and Coles, 2002). Error processing dysfunction has been demonstrated in several psychiatric conditions, including schizophrenia (Becerril et al., 2011; Mathalon et al., 2009; Morris et al., 2008), depression (Chiu and Deldin, 2007; Steele et al., 2004; Tucker et al., 2003) and a range of drug dependencies (Connolly et al., 2012; Easdon et al., 2005; Forman et al., 2004; Li et al., 2010). In all these conditions, the dysfunction is characterised by hypoactivity in the errorrelated network, most consistently in the dorsal anterior cingulate gyrus (dACC). While relatively few studies have investigated error processing in chronic cannabis users (see Spronk et al., 2011 for a study of the acute effects on non-users), the typical pattern of hypoactivity in the AACC (along with other key error-related regions such as the insula) has recently been demonstrated (Hester et al., 2009). Thus, there is evidence to suggest that performance monitoring is impaired in chronic cannabis users, although the consequence for adaptive post-error behaviour remains unclear.

The dorsal anterior cingulate cortex (dACC; Fitzgerald et al., 2010; Ridderinkhof, 2004; Ullsperger and von Cramon, 2003) and mesial temporal memory structures (Squire et al., 2004) have been consistently implicated in reinforcement learning. The magnitude of dACC activation has been associated with adaptive changes following an error. Greater dACC activation has been associated with individuals slowing their response following the error (Debener et al., 2005; Garavan et al., 2002), with post-error slowing thought to reflect cautiousness and to be linked with future behavioural changes ("learning"). Research in drug-dependent populations has consistently linked error-related hypoactivity in the dACC with a lack of post-error slowing (Forman et al., 2004; Franken et al., 2007; Hester et al., 2007), though the relationship between slowing and adaptive behaviour is often difficult to 
demonstrate with some cognitive paradigms. Similarly, the dACC has also been implicated in other adaptive behaviours (Agam et al., 2011), with greater activation in the dACC associated with making fewer commission errors (Ghahremani et al., 2010; Polli et al., 2008). Chronic cannabis using samples have also shown hypoactive dACC activity during dysfunctional control performance (Gruber and Yurgelun-Todd, 2005; Wesley et al., 2011) that was associated with poorer overall task performance. Recent research has demonstrated that error-related dACC activity predicts learning from errors, despite a substantial delay between the error and the opportunity to correct the error (Hester et al., 2008, 2010). In particular, the relationship between error-related activity in the dorsal ACC and re-encoding related activity in the hippocampus was particularly critical to successful learning from errors.

Error processing dysfunction in drug abusing populations has been found to increase the likelihood of drug-seeking behaviour and interfere with a user's capacity to assimilate and participate in rehabilitation programs that have an educative and cognitive emphasis (Sofuoglu et al., 2010; Verdejo-García et al., 2004). The purpose of the current study was to examine error processing in chronic cannabis users by utilising a combined neuroimaging and behavioural approach. Given the previous findings of error-related hypoactivity in cannabis users (Hester et al., 2009), we sought to examine the relationship between errorrelated dACC activity and learning from errors in cannabis users and controls, using a paired associate learning task (2008).

We hypothesised that chronic cannabis users would have poorer recall on the paired associate learning task, with significantly lower error correction rate when taking into account the poorer initial performance. It was also hypothesised that chronic cannabis users would have hypoactivity in the $\mathrm{dACC}$ and hippocampus during error processing in comparison to controls, and that this hypoactivity would be associated with poorer error correction rate.

\section{METHODS}

\subsection{Sample and Participant Selection}

Fifteen chronic cannabis users (four females, mean age $=22.40$ years, $S D=4.29$, range $=$ 18-33) and 15 control participants (two females, mean age $=23.27$ years, $S D=3.67$, range = 19-33) were recruited via leaflet advertising at Trinity College Dublin, Dublin, Ireland. Written informed consent was obtained from all participants following complete description of the study. Groups were matched for educational attainment, $t(28)=1.23, p=.229$, and estimated pre-morbid IQ, via the National Adult Reading Test (NART; Nelson, 1982), $t(28)$ $=-0.60, p=.555$ (see Table 1). A semi-structured interview was used to screen participants for past or present history of psychiatric or neurological illness. Participants were interviewed using a self-report form that screened for a history of diagnosed psychiatric or neurological illness, including prompts for the 20 most commonly diagnosed conditions in the age-group sampled (college students). Participants were also asked to report any current undiagnosed symptoms that they were experiencing, which were followed up with verbal questions to clarify the type, intensity and duration of symptoms. Information pertaining to any form of treatment (counselling, psychological, psychiatric), past or present, was 
carefully detailed, with any potential participant describing any major life-time psychiatric event or brain injury (e.g., head trauma resulting in a loss of consciousness, seizure or stroke) considered ineligible for the study. Participants were also considered ineligible if they reported any familial psychiatric history (i.e., sibling, parent or grandparent).

In order to screen for past or current abuse of other substances, all participants completed the inventories of drug use subsection of the Addiction Severity Index Lite (Clinical Factors version) questionnaire (McLellan et al., 1999). Prospective participants from either group were considered ineligible if they reported concurrent or past dependence on other drugs (including tobacco and alcohol). Information concerning alcohol and cannabis use in each participant was indexed in number of years (lifetime) and occasions of recent use (last 30 days) and is presented in Table 1.

In order to be eligible for participation in this study, participants in the cannabis group were required to have regularly consumed cannabis (5-7 days/week) for the previous two years and to have smoked a minimum of 500 joints in their lifetime. All cannabis users provided a positive urine sample for $\Delta^{9}$-tetrahydrocannabinol $\left(\Delta^{9} \mathrm{THC}\right)$ before scanning, with an additional screening for other confounding drug use (Cozart RapiScan, Abingdon, UK) taking place. Control participants were also urine tested for $\Delta^{9} \mathrm{THC}$ and other drugs. Prospective participants with a past or present diagnosis (or self-reported symptoms consistent with a current diagnosis) of psychiatric or neurological illness were excluded. Participants providing positive tests for drugs other than cannabis (cannabis group only) were excluded, and all participants provided a breath test $0 \%$ blood alcohol concentration reading before the beginning of the cognitive testing.

\subsection{Experimental protocols}

A paired associate learning task (Hester et al., 2008), consisting of an array of locationnumber associations that were to be learned by participants, was administered (Figure 1). All aspects of stimulus delivery and response recording were controlled by E-Prime software (version 1.1; Psychology Software Tools), running on a laptop PC (Celeron 2-Ghz, 128MB Nvidia video card) that was interfaced with the magnetic resonance (MR) scanner during fMRI acquisition. The task began with an encoding phase in which eight locations designated as light grey squares were presented simultaneously on a dark grey background. The locations of the squares on the background were selected in a quasi-random fashion from an $8 \times 8$ matrix, with two locations randomly chosen from each of the four quadrants on the display.

At the commencement of the encoding phase, each location in turn had superimposed upon it a two-digit number. The number remained visible for two seconds, and was followed by an inter-stimulus interval of one second. The digits of each number consisted of 1,2,3 or 4, and participants identified the number by entering each digit using the appropriate buttons on a pair of MR-compatible response boxes (Fibre-Optic response pads, Current Designs, Philadelphia, PA, USA). Two digit numbers were used to reduce the probability of guessing the correct answer to $6 \%$. The encoding phase lasted 24 seconds in total, and was followed by a 6 -second interval prior to the start of the recall phase.

Drug Alcohol Depend. Author manuscript; available in PMC 2016 October 01. 
Following the encoding phase in which numbers were shown for each of the eight locations, a series of recall trials was presented. During a recall trial, one of the eight locations was highlighted in yellow, cueing the participant to respond with the two-digit number associated with that location. Participants were required to respond within two seconds, after which feedback was presented for one second. Feedback indicated if the response was correct and also provided the correct number. The location square turned blue to indicate a correct response, or red to indicate an incorrect response, and the correct number was shown upon the coloured background. Therefore, the participant received immediate response feedback and had another opportunity to encode the correct response. Following feedback, a variable delay of three to nine seconds was introduced prior to the next recall trial, during which all 8 location squares remained on the screen. This variable delay had the effect of jittering the onset of each recall trial, which is necessary for event-related fMRI designs in which blood oxygen-level dependent (BOLD) changes are modelled for single trials. Each location in the array was highlighted once, before being highlighted a second time, all in different pseudo-random orders. We ensured that the two presentations of any trial were between 7-9 trials. This created two rounds of eight recall trials within each block. Eight blocks of the encoding/recall cycle were administered to each participant, with each block involving a different array of locations and two-digit numbers. No location in the array was used more than once throughout the eight runs, and the two digit numbers were not repeated on consecutive blocks.

\subsection{Data Analysis}

The fMRI data acquisition and pre-processing analysis is detailed in the supplementary materials ${ }^{1}$. A mixed regression group fMRI analysis was employed comprising multiple regressors. Behavioural data from each participant were used to categorise the recall events into successful responses, corrected errors, and repeated errors. Errors were classified in this way according to the response made on the subsequent presentation of the same locationnumber pair (see Figure 2). An incorrect response that was followed by another incorrect response for the same location in the subsequent round was classed as a 'repeated error', whereas an error that was followed by a correct response in the following round was classed as a 'corrected error'. Errors in the second round of presentations could therefore not be included in this analysis because they did not precede another attempt at recall.

The primary comparison of interest was to compare differences in activation between corrected and repeated errors. The activation clusters from whole-brain analyses of the feedback epoch for errors were used for the purposes of a functionally-defined region of interest (ROI) analysis. The events of interest for the group map were the errors from round 1 of recall, of which participants made, on average, 44 errors (range $=32-58$ ). A second analysis was then performed, which entered additional regressors into the deconvolution process to separately estimate activity related to the two types of error-related events (corrected and repeated errors), relative to baseline. Group activation maps for event-type (corrected errors, repeated errors) were determined with one-sample t-tests against the null hypothesis of zero event-related activation changes (i.e., no change relative to baseline).

\footnotetext{
${ }^{1}$ Supplementary material can be found by accessing the online version of this paper at http://dx.doi.org and by entering doi:...
} 
Significant voxels passed a voxelwise statistical threshold $(t=4.31, p \leq .001)$ and were required to be part of a larger $142 \mu$ l cluster of contiguous significant voxels. The mean activation for clusters in the functionally defined error map was then calculated for the purposes of an ROI analysis, deriving mean activation levels for corrected errors and repeated errors for each participant. These estimates were compared using repeatedmeasures $t$-tests, corrected for the number of ROIs via a modified Bonferroni procedure for multiple comparisons (Keppel, 1999). See S1 for details of the image acquisition and analysis process.

\section{RESULTS}

\subsection{Behavioural Results}

Accurate recall of number-location associations during the first round differed significantly between groups, with controls demonstrating more accurate performance $(M=35.8 . \%, S D=$ $8.9 \%)$ than the cannabis group $(M=25.9 \%, S D=11.3 \%)$, unbiased Hedge's $g$ (effect size) $=$ $0.78,95 \%$ CI $[0.02,1.75], t(28)=2.66, p=.013$. The percentage of correct responses in round 2 was significantly different between groups, with the control group correctly recalling more number-location associations $(M=51.5 \%, S D=13.3 \%)$ than the cannabis group $(M=34.4 \%, S D=17.8 \%), g=1.06,95 \%$ CI $[0.29,1.83], t(28)=2.98, p=.006$.

To examine the rate of learning from errors, an error correction rate was calculated by dividing the number of recall errors in round 1 that were subsequently corrected in round 2, by the total number of errors made in round 1 . The control group demonstrated a higher rate of error correction than the cannabis group $(M=38.2 \%$ and $26.9 \%, S D=13.8 \%$ and $14.9 \%$, respectively), $g=0.76,95 \%$ CI $[0.02,1.51], t(28)=2.15, p=.040$. The rate of corrected errors for both groups is displayed in Figure 3.

There were no differences between the control and CCU groups in response times for error responses, $F(1,28)=2.52, p=.12$, or correct responses, $F(1,28)=0.97, p=.33$.

The cannabis use variables were combined into a single composite score to minimise statistical power issues (see pg 4 of supplementary material for method ${ }^{2}$ ) and correlations between the composite cannabis use score and performance indices of interest are displayed in Table 2. There was a significant and negative association between the number of correct first round responses and the composite cannabis score, with higher ratings of cannabis use habits associated with poorer recall accuracy. This significant negative correlation was also observed when the first and second round recall accuracy rates were converted into a percentage of overall responses. Neither the number of corrected errors nor the error correction percentage was associated with the cannabis use composite score. However, repeated errors were significantly associated with the cannabis use composite score: higher cannabis use was associated with more repeated errors. Overall recall reaction time and reaction time for error responses each had a significant positive correlation with cannabis use, as participants with higher composite cannabis use scores responded more slowly. These associations were not evident for correct responses.

\footnotetext{
${ }^{2}$ Supplementary material can be found by accessing the online version of this paper at http://dx.doi.org and by entering doi:...
} 


\subsection{Imaging Data}

Using the functionally defined clusters from the error-related activity map, a 2 group $\mathrm{x} 2$ error type (corrected, repeated) ROI analysis was performed. A main effect of error type was found in several regions (see Table 3), including the dACC (the centre-of-mass for this cluster of activity was located at MNI coordinates: $x=7 ; y=13 ; z=52$ ) and left hippocampus (MNI coordinates: $x=-31 ; y=-22 ; z=-13$ ). Corrected errors were associated with significantly higher levels of BOLD activity in comparison to repeated errors in these functionally defined clusters. Activity in several other regions also showed higher levels of BOLD activity for corrected errors compared to repeated errors, including right putamen, right middle occipital gyrus, and left inferior frontal gyrus (pars opercularis). Only the right posterior cingulate cortex was associated with higher level of BOLD activity for repeated errors compared to corrected errors.

A significant main effect of group was found in a range of error-related regions, with significantly higher levels of BOLD activity for the control group compared to the cannabis user group in the right superior parietal lobule, right inferior parietal lobule, bilateral dACC, right anterior cingulate, left inferior temporal gyrus, left inferior parietal lobule, left hippocampus, left middle cingulate cortex, right thalamus, left putamen, left postcentral gyrus, left thalamus, and left supramarginal gyrus (see Table 4). There were no error-related ROIs where cannabis users had higher levels of BOLD activity than controls during errorrelated activity.

A test of the interaction between group (cannabis users, controls) and error type (corrected, repeated errors) was conducted on the BOLD activity for each ROI. Two clusters in the dACC showed a significant interaction effect, as did the left hippocampus. The pattern of activity in these three ROIs indicated that controls had greater BOLD activity during corrected errors than during repeated errors in comparison to cannabis users, who did not demonstrate a significant difference between the two error types (see Figure 4). The pattern in the left dorsal ACC (see Figure 4.B.1) suggests that the cannabis-using group showed an opposing pattern of greater BOLD activity for repeated in comparison to corrected errors, however this difference was not significant. Other ROIs that indicated a group by error type interaction in levels of BOLD activity are listed in Table 5. Briefly, in the following regions, controls demonstrated greater levels of activity during corrected errors than repeated errors, whereas cannabis users did not demonstrate a significant difference in activation between error types: left inferior temporal gyrus, right thalamus ( $x=18 ; y=-25 ; z=-10)$, right temporal pole, left superior occipital gyrus, left putamen, and left thalamus. Interestingly, a second cluster in the right thalamus $(\mathrm{x}=6 ; \mathrm{y}=-23 ; \mathrm{z}=3)$ showed the opposite pattern with greater activation during corrected errors compared to repeated errors for the cannabis group, whereas the control group did not show differential activation as a function of error type. Finally, in comparison to cannabis users, the control group showed significantly greater activation of the right inferior parietal lobe and left supramarginal gyrus during corrected errors only.

There were no significant correlations between any ROIs and the cannabis use composite score at the $p<.05$ level. Although non-significant, the effect sizes of the correlations 
between the cannabis use composite scores and the ROIs ranged between .01 and .44.

Correlations between individual cannabis use measures (including recency of use measures) and ROI activity did not identify significant associations after correcting for the high number of comparisons.

\section{DISCUSSION}

The aims of this study were (1) to investigate whether chronic cannabis users had impaired learning from errors in comparison to a matched control sample, and (2) to determine which brain regions were associated with poorer learning from errors. Consistent with our hypothesis, cannabis users showed significantly poorer recall accuracy and a lower correction rate (percentage of errors corrected on subsequent trials) on a paired associate learning task when compared with healthy controls. The diminished error correction rate shown by the cannabis group was associated with hypoactive error-related BOLD responses, when compared to control participants, in the bilateral dACC, left hippocampus, left putamen, bilateral thalami, and bilateral inferior parietal lobes. Specifically, the cannabis group's error-related response in these regions did not significantly differentiate corrected from repeated errors, whereas control participants' response showed a significant BOLD increase during corrected errors. These findings are commensurate with previous literature, where higher levels of BOLD activity in the dACC and hippocampus predicted greater learning from errors (Hester et al., 2008). As further support for the dACC being error specific, we did not find the same pattern of activity in the pre-supplementary motor area (adjacent to the dACC), which is argued to be related to preparation for motor activity posterror (Nachev et al., 2007).

The finding that cannabis users have compromised learning is consistent with studies examining learning from errors in a range of substance-using populations and psychiatric disorders (Fitzgerald et al., 2005; Forman et al., 2004; Franken et al., 2007; Hester et al., 2007; Morris et al., 2008). Given that significant memory impairments are not readily discerned in individuals with less than a decade's history of heavy cannabis use (Solowij et al., 2002), the current result in conjunction with the commensurate literature in other substance-using populations strongly suggests that failing to modify behaviour following a mistake is a consistent feature of chronic substance abuse. Chronic cannabis using samples have previously shown hypoactive dACC activity during dysfunctional control performance (Gruber and Yurgelun-Todd, 2005; Wesley et al., 2011) that was associated with poorer overall task adaptation. Because failing to learn from negative outcomes is a required DSM criterion for a diagnosis of substance abuse or dependence (American Psychiatric Association, 2000), a clinical implication of these findings is that impairments in learning from errors may be associated with poor treatment outcomes, as has recently been found in other drug-using populations (Luo et al., 2013; Marhe et al., 2013).

Taken together, the behavioural and neurobiological findings are consistent with the prediction from Holroyd and Coles' (2002) theory that mesencephalic dopamine activity is utilised by the dACC to reinforce adaptive behaviours and modify unsuccessful performance (where the outcome is worse than expected). In the healthy controls, the level of BOLD activity in the dACC was greater when encoding feedback for errors that subsequently 
corrected; that is, the controls activated the dACC significantly more when adjusting their unsuccessful performance. This is consistent with previous studies in healthy controls (Hester et al., 2008, 2010). Our data suggest that one important contributor to cannabis users' learning impairments may be underpinned by neurobiological abnormalities in the error processing network.

It is unclear the extent to which this error-related impairment and associated dACC hypoactivity reflects an abnormality that predates and possibly contributes to the conversion from occasional cannabis use to chronic use, or whether the chronic use of cannabis has contributed to the learning from error impairment. There is some evidence from animal models of differences in sensitivity to negative feedback between animals that transition from occasional use to higher use than those that do not (Dalley et al. 2011). For example, in comparison to rats low in impulsivity, highly impulsive rats continued to demonstrate cocaine-seeking behaviours despite receiving negative feedback (electric shocks) during drug exposure, suggesting that high impulsivity traits and low sensitivity to negative feedback are premorbid factors that contribute to the transition to problematic drug use (Economidou et al., 2009). On the other hand, other animal studies have shown that the increasing use of substances such as cocaine and heroin causes neurobiological changes to the reward circuitry (including the dopaminergic system), which in turn contributes to the diminished sensitivity to negative feedback (Koob et al., 2004). Human studies have shown that this diminished sensitivity to negative feedback predicts poorer learning (Frank et al., 2005). Furthermore, at-risk adolescent children of substance use disorder parents demonstrate diminished error-related negativities that were not reflected in behavioural tasks, suggesting that abnormal error processing may represent a vulnerability factor for developing substance use disorders (Euser et al., 2012). Therefore, there is some evidence for diminished learning from errors being both a pre-existing risk factor for developing a substance use disorder and a consequence of increasing substance use; however, this has yet to be fully explored in the cannabis-using population.

Previous findings of neuropsychological impairments found within chronic cannabis-using populations have been argued to represent recent cannabis use intoxication and/or withdrawal symptoms, rather than associated with cortical changes (e.g., Accordino, 2006). Performance in the present study does not appear to have been affected by these confounds, with post hoc examination of the data finding no association between recency of use and error-related activity or recall performance. Similarly, all participants in the cannabis using group abstained for at least 12 hours prior to scanning (101 hours on average), which is outside the typical window of acute effects of cannabis on recall performance (Grotenhermen, 2003). The possibility of neuropsychological impairments being related to the cannabis withdrawal syndrome appears unlikely as the peak effects occur two-to-six days post-cessation (Budney et al., 2004), consistent with our CCU participants low selfreported withdrawal symptom scores prior to testing.

By using a paired associate learning task that provides the opportunity to immediately reencode the correct response following a mistake, our data indicate that cannabis users have compromised learning abilities. Their poorer post-error recall performance was related to diminished neural activity in regions typically associated with error processing, particularly 
the dACC and hippocampus. Cannabis users' failure to learn from their errors is consistent with the clinical profile of perseverative behaviours associated with chronic cannabis use. These findings have implications for understanding continued substance use despite the associated negative consequences, and may assist in developing therapeutic interventions that modifies treatment to account for the compromised cognitive learning abilities of this population.

\section{Supplementary Material}

Refer to Web version on PubMed Central for supplementary material.

\section{Acknowledgments}

Role of Funding Sources: This study was supported by USPHS grant from the National Institute on Drug Abuse: DA01865-01, Australian Research Council Future Fellowship (RH) FT110100088. Neither funding agency had any further role in the study design; in the collection, analysis and interpretation of data; in the writing of the report; or in the decision to submit the paper for publication.

The assistance of Gloria Roberts, Gina Joue and Ella McCabe are gratefully acknowledged.

\section{References}

Accordino M. Neuropsychological deficits in long-term frequent cannabis users: to the Editor. Neurology. 2006; 67:1902. [PubMed: 17130442]

Agam Y, Hämäläinen MS, Lee AKC, Dyckman KA, Friedman JS, Isom M, Makris N, Manoach DS. Multimodal neuroimaging dissociates hemodynamic and electrophysiological correlates of error processing. Proc Natl Acad Sci U S A. 2011; 108:17556-17561. [PubMed: 21969565]

American Psychiatric Association. Diagnostic and Statistical Manual of Mental Disorders. APA; Washington, DC: 2000.

Becerril KE, Repovs G, Barch DM. Error processing network dynamics in schizophrenia. Neuroimage. 2011; 54:1495-1505. [PubMed: 20883800]

Budney AJ, Hughes JR, Moore BA, Vandrey R. Review of the validity and significance of cannabis withdrawal syndrome. Am J Psychiatry. 2004; 161:1967-1977. [PubMed: 15514394]

Chiu PH, Deldin PJ. Neural evidence for enhanced error detection in major depressive disorder. Am J Psychiatry. 2007; 164:608-616. [PubMed: 17403974]

Connolly CG, Foxe JJ, Nierenberg J, Shpaner M, Garavan H. The neurobiology of cognitive control in successful cocaine abstinence. Drug Alcohol Depend. 2012; 121:45-53. [PubMed: 21885214]

Crean RD, Tapert SF, Minassian A, MacDonald K, Crane NA, Mason BJ. Effects of chronic, heavy cannabis use on executive functions. J Addict Med. 2011; 5:9-15. [PubMed: 21643485]

Debener S, Ullsperger M, Siegel M, Fiehler K, von Cramon DY, Engel AK. Trial-by-trial coupling of concurrent electroencephalogram and functional magnetic resonance imaging identifies the dynamics of performance monitoring. J Neurosci. 2005; 25:11730-11737. [PubMed: 16354931]

Easdon C, Izenberg A, Armilio ML, Yu H, Alain C. Alcohol consumption impairs stimulus- and errorrelated processing during a Go/No-Go Task. Cogn Brain Res. 2005; 25:873-883.

Economidou D, Pelloux Y, Robbins TW, Dalley JW, Everitt BJ. High impulsivity predicts relapse to cocaine-seeking after punishment-induced abstinence. Biol Psychiatry. 2009; 65:851-856. [PubMed: 19181308]

Euser AS, Evans BE, Greaves-Lord K, Huizink AC, Franken IH. Diminished error-related brain activity as a promising endophenotype for substance-use disorders: evidence from high-risk offspring. Addict Biol. 2012; 18:970-984. [PubMed: 23145495]

Fitzgerald KD, Perkins SC, Angstadt M, Johnson T, Stern ER, Welsh RC, Taylor SF. The development of performance-monitoring function in the posterior medial frontal cortex. Neuroimage. 2010; 49:3463-3473. [PubMed: 19913101]

Drug Alcohol Depend. Author manuscript; available in PMC 2016 October 01. 
Fitzgerald KD, Welsh RC, Gehring WJ, Abelson JL, Himle JA, Liberzon I, Taylor SF. Error-related hyperactivity of the anterior cingulate cortex in obsessive-compulsive disorder. Biol Psychiatry. 2005; 57:287-294. [PubMed: 15691530]

Forman SD, Dougherty GG, Casey BJ, Siegle GJ, Braver TS, Barch DM, Stenger VA, Wick-Hull C, Pisarov LA, Lorensen E. Opiate addicts lack error-dependent activation of rostral anterior cingulate. Biol Psychiatry. 2004; 55:531-537. [PubMed: 15023582]

Frank MJ, Woroch BS, Curran T. Error-related negativity predicts reinforcement learning and conflict biases. Neuron. 2005; 47:495-501. [PubMed: 16102533]

Franken IHA, van Strien JW, Franzek EJ, van de Wetering BJ. Error-processing deficits in patients with cocaine dependence. Biol Psychiatry. 2007; 75:45-51.

Garavan H, Ross TJ, Murphy K, Roche RA, Stein EA. Dissociable executive functions in the dynamic control of behavior: inhibition, error detection, and correction. Neuroimage. 2002; 17:1820-1829. [PubMed: 12498755]

Ghahremani DG, Monterosso J, Jentsch JD, Bilder RM, Poldrack RA. Neural components underlying behavioral flexibility in human reversal learning. Cereb Cortex. 2010; 20:1843-1852. [PubMed: 19915091]

Grant I, Gonzalez R, Carey CL, Natarajan L, Wolfson T. Non-acute (residual) neurocognitive effects of cannabis use: a meta-analytic study. J Int Neuropsychol Soc. 2002; 9:679-689. [PubMed: 12901774]

Grotenhermen F. Pharmacokinetics and pharmacodynamics of cannabinoids. Clin Pharmacokinet. 2003; 42:327-360. [PubMed: 12648025]

Gruber SA, Yurgelun-Todd DA. Neuroimaging of marijuana smokers during inhibitory processing: a pilot investigation. Brain Res Cogn Brain Res. 2005; 23:107-118. [PubMed: 15795138]

Hester R, Barre N, Mattingley J, Foxe J. Avoiding another mistake: error and posterror neural activity associated with adaptive posterror behavior change. Cogn Affect Behav Neurosci. 2007; 7:317326. [PubMed: 18189005]

Hester R, Barre N, Murphy K, Silk T, Mattingley JB. Human medial frontal cortex activity predicts learning from errors. Cereb Cortex. 2008; 18:1933-1940. [PubMed: 18063560]

Hester R, Murphy K, Brown F. Punishing an error improves learning: the influence of punishment magnitude on error-related neural activity and subsequent learning. J Neurosci. 2010; 30:1560015607. [PubMed: 21084615]

Hester R, Nestor L, Garavan H. Impaired error awareness and anterior cingulate cortex hypoactivity in chronic cannabis users. Neuropsychopharmacology. 2009; 34:2450-2458. [PubMed: 19553917]

Holroyd CB, Coles MGH. The neural basis of human error processing: reinforcement learning, dopamine, and the error-related negativity. Psychol Rev. 2002; 109:679-709. [PubMed: 12374324]

Kalivas PW, Volkow ND. The neural basis of addiction: a pathology of motivation and choice. Am J Psychiatry. 2005; 162:1403-1413. [PubMed: 16055761]

Keppel, G. Design and Analysis: A Researcher's Handbook. Prentice Hall; Englewood Cliffs, NJ: 1999.

Koob GF, Ahmed SH, Boutrel B, Chen SA, Kenny PJ, Markou A, O'Dell LE, Parsons LH, Sanna PP. Neurobiological mechanisms in the transition from drug use to drug dependence. Neurosci Biobehav Rev. 2004; 27:739-749. [PubMed: 15019424]

Li CSR, Luo X, Sinha R, Rounsaville BJ, Carroll KM, Malison RT, Ding YS, Zhang S, Ide JS. Increased error-related thalamic activity during early compared to late cocaine abstinence. Drug Alcohol Depend. 2010; 109:181-189. [PubMed: 20163923]

Luo X, Zhang S, Hu S, Bednarski SR, Erdman E, Farr OM, Hong KI, Sinha R, Mazure CM, Li CSR. Error processing and gender-shared and -specific neural predictors of relapse in cocaine dependence. Brain. 2013; 136:1231-1244. [PubMed: 23485852]

Marhe R, Luijten M, van de Wetering BJM, Smits M, Franken IHA. Individual differences in anterior cingulate activation associated with attentional bias predict cocaine use after treatment. Neuropsychopharmacology. 2013; 38:1085-1093. [PubMed: 23303067]

Mathalon DH, Jorgensen KW, Roach BJ, Ford JM. Error detection failures in schizophrenia: ERPs and fMRI. Int J Psychophysiol. 2009; 73:109-117. [PubMed: 19414043] 
McLellan, AT.; Cacciola, J.; Carise, D.; Coyne, TH. The Addiction Severity Index-Lite: Clinical Factors Version (ASI-Lite-CF). The Treatment Research Institute; Philadelphia, PA: 1999.

Messinis L, Kyprianidou A, Malefaki S, Papathanasopoulos P. Neuropsychological deficits in longterm frequent cannabis users. Neurology. 2006; 66:737-739. [PubMed: 16534113]

Morris SE, Heerey E, Gold J, Holroyd CB. Learning-related changes in brain activity following errors and performance feedback in schizophrenia. Schizophr Res. 2008; 99:274-285. [PubMed: 17889510]

Nachev P, Wydell H, O’Neill K, Husain M, Kennard C. The role of the pre-supplementary motor area in the control of action. Neuroimage. 2007; 36:T155-163. [PubMed: 17499162]

Nelson, HE. National Adult Reading Test: Test Manual. NFER-Nelson; Windsor, UK: 1982.

Polli FE, Barton JJS, Thakkar KN, Greve DN, Goff DC, Rauch SL, Manoach DS. Reduced errorrelated activation in two anterior cingulate circuits is related to impaired performance in schizophrenia. Brain. 2008; 131:971-986. [PubMed: 18158315]

Ranganathan M, D'Souza DC. The acute effects of cannabinoids on memory in humans: a review. Psychopharmacology (Berl). 2006; 188:425-444. [PubMed: 17019571]

Ridderinkhof KR. The role of the medial frontal cortex in cognitive control. Science. 2004; 306:443447. [PubMed: 15486290]

Sofuoglu M, Sugarman DE, Carroll KM. Cognitive function as an emerging treatment target for marijuana addiction. Exp Clin Psychopharmacol. 2010; 18:109-119. [PubMed: 20384422]

Solowij N, Battisti R. The chronic effects of cannabis on memory in humans: a review. Curr Drug Abuse Rev. 2008; 1:81-98. [PubMed: 19630708]

Solowij N, Stephens RS, Roffman RA, Babor T, Kadden R, Miller M, Christiansen K, McRee B, Vendetti J. Cognitive functioning of long-term heavy cannabis users seeking treatment. JAMA. 2002; 287:1123-1131. [PubMed: 11879109]

Spronk D, Dumont GJH, Verkes RJ, de Bruijn ERA. Acute effects of delta-9-tetrahydrocannabinol on performance monitoring in healthy volunteers. Front Behav Neurosci. 2011; 5:1-11. [PubMed: 21267359]

Squire LR, Stark CEL, Clark RE. The medial temporal lobe. Annu Rev Neurosci. 2004; 27:279-306. [PubMed: 15217334]

Steele JD, Meyer M, Ebmeier KP. Neural predictive error signal correlates with depressive illness severity in a game paradigm. Neuroimage. 2004; 23:269-280. [PubMed: 15325374]

Tucker DM, Luu P, Frishkoff G, Quiring J, Poulsen C. Frontolimbic response to negative feedback in clinical depression. J Abnorm Psychol. 2003; 112:667-678. [PubMed: 14674878]

Ullsperger M, von Cramon DY. Error monitoring using external feedback: specific roles of the habenular complex, the reward system, and the cingulate motor area revealed by functional magnetic resonance imaging. J Neurosci. 2003; 23:4308-4314. [PubMed: 12764119]

Verdejo-García A, López-Torrecillas F, Giménez CO, Pérez-García M. Clinical implications and methodological challenges in the study of the neuropsychological correlates of cannabis, stimulant, and opioid abuse. Neuropsychol Rev. 2004; 14:1-41. [PubMed: 15260137]

Wesley MJ, Hanlon CA, Porrino LJ. Poor decision-making by chronic marijuana users is associated with decreased functional responsiveness to negative consequences. Psychiatry Res. 2011; 191:51-59. [PubMed: 21145211] 


\section{Highlights}

- Cannabis users performed an associative learning task during fMRI data collection

- Cannabis users compared to controls showed poorer recall performance and error correction rate

- Cannabis users failure to adapt performance was associated with hypoactivity in error-related dACC and hippocampal regions 


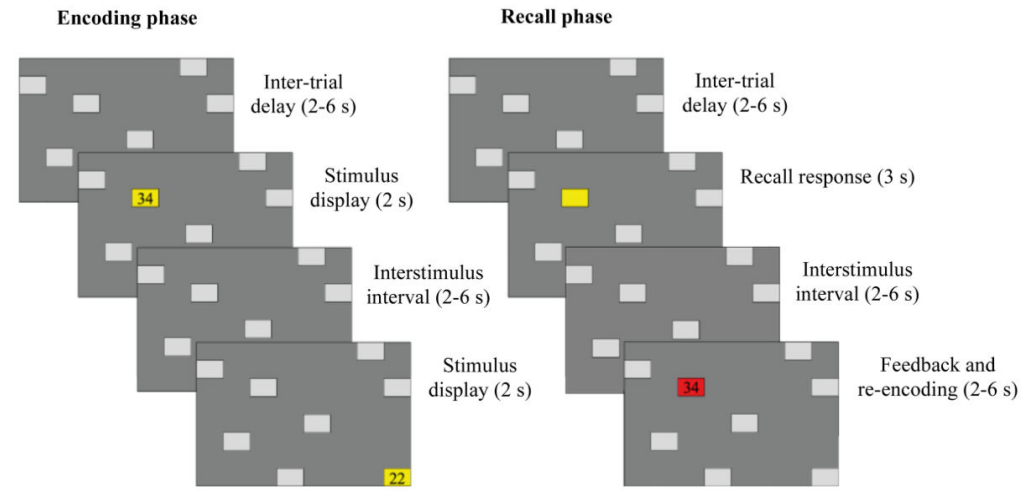

Figure 1.

The paired associate learning task used in the present study, represented by the screen transitions for the encoding and recall phases of the task. Each block of trials began with an encoding phase that presented the two-digit number associated with each location and an inter-trial interval display. All eight number-location associations were presented once during the encoding phase and were immediately followed by the recall phase. A single trial in the recall phase began by highlighting a location in yellow to cue the participant to respond with the two-digit number they associated with the location. Following a variable interstimulus delay, a simultaneous feedback and re-encoding opportunity was provided that consisted of presenting the accuracy of the response (location turned red for an error, blue for correct; see Figure 2) with the actual number associated with the location superimposed upon the coloured square. The number was displayed regardless of prior recall accuracy. 


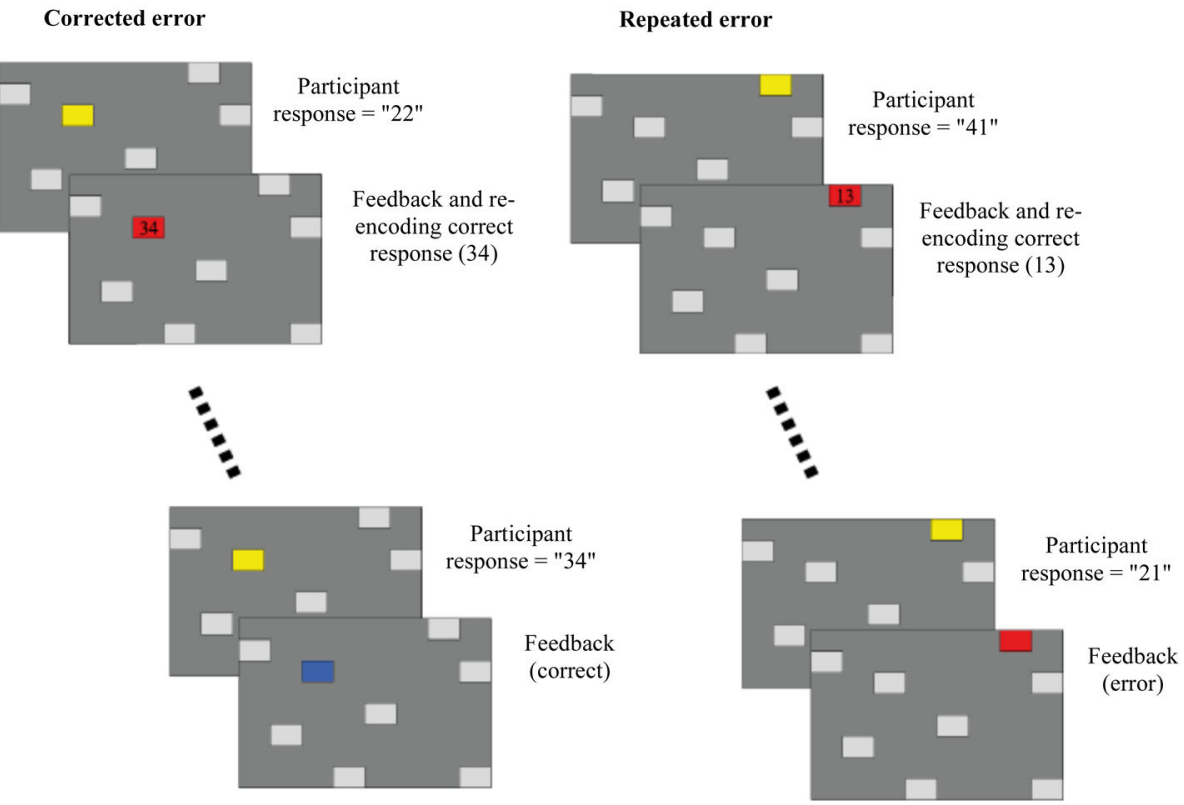

Figure 2.

Method used to classify corrected and repeated errors. Feedback for a participant's response involved presentation of the correct number on either a red background (indicating an error) or a blue background (indicating an accurate recall response). Categorisation as either a corrected or repeated error was determined by the participant's performance for the same trial during the next round. In the corrected error example, the participant incorrectly recalled the digits associated with the upper left location (responding with 22 rather than 34) during round 1, but correctly recalled these digits during round 2 - and so the initial round 1 error is categorised as a corrected error. In the repeated error example, the participant incorrectly responded to the presentation of the top right location during both round 1 and 2, and therefore the initial error is categorised as a repeated error. Black squares represent the intervening trials. 


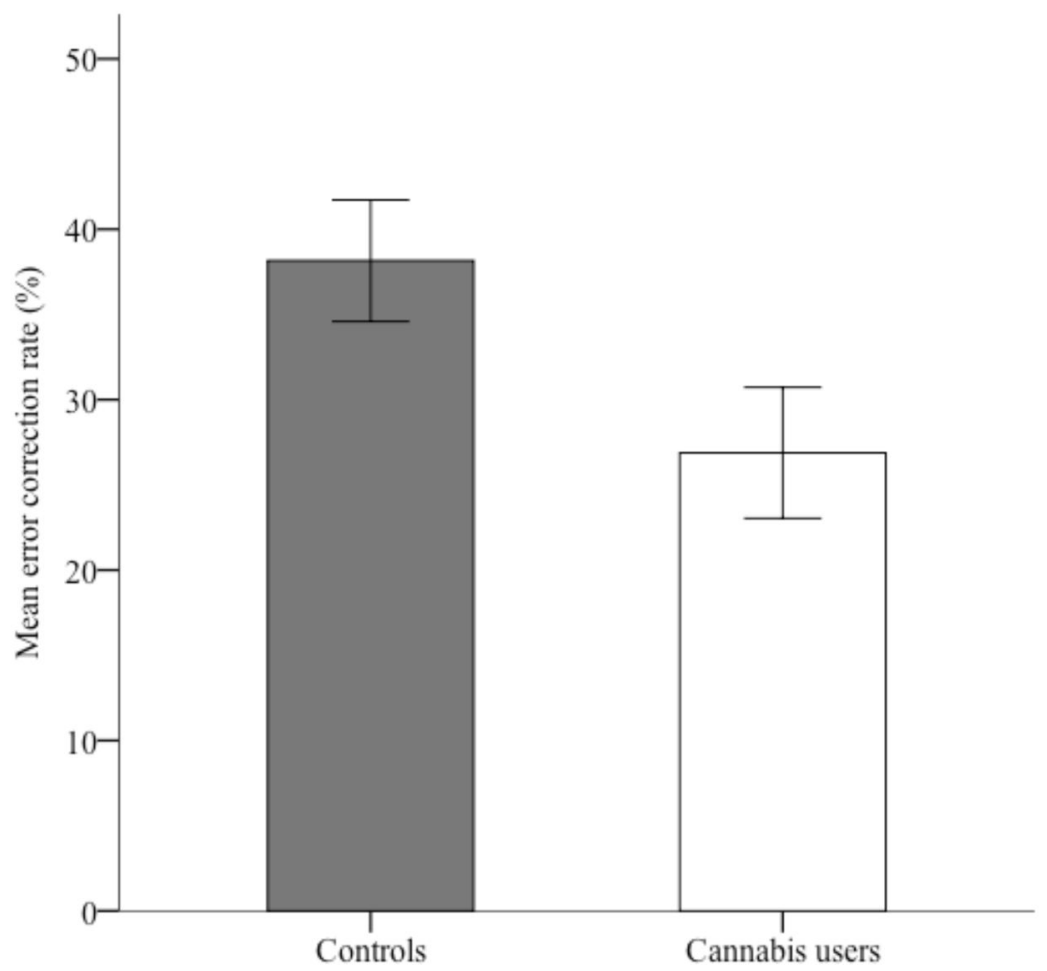

Figure 3.

Mean recall error correction rates (expressed as a percentage of total errors in round 1 that were corrected in round 2) for control and cannabis groups. Error bars represent standard error of the mean. 

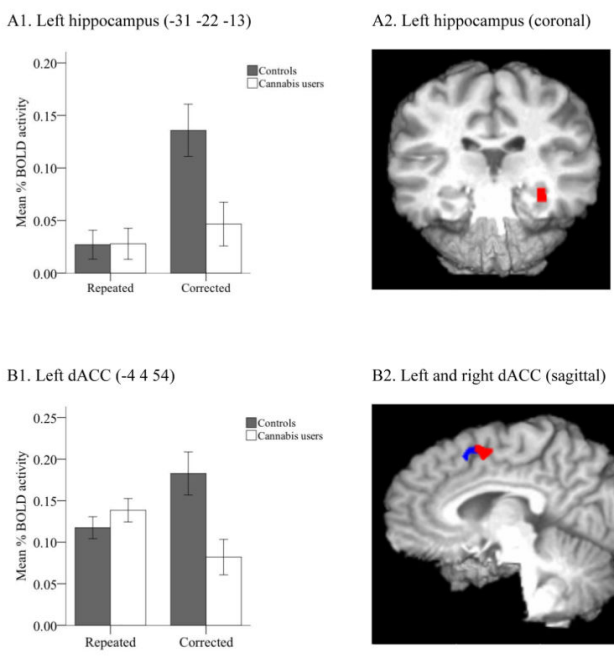

B2. Left and right dACC (sagittal)

Cl. Right dACC ( 713 52)
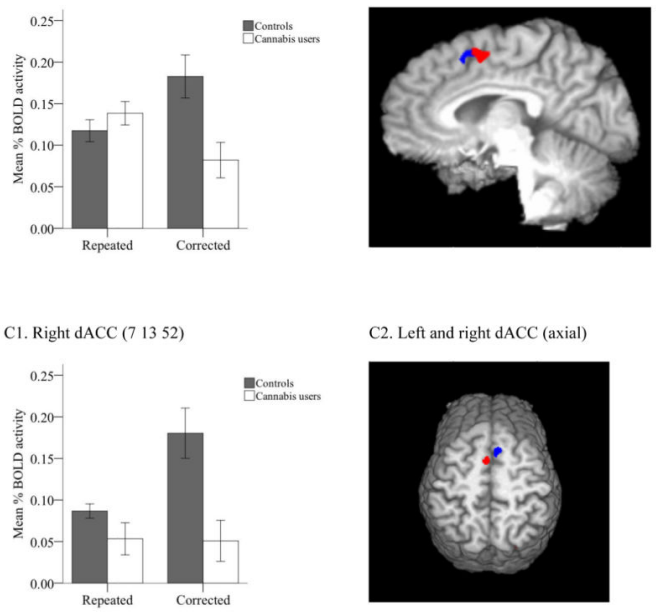

C2. Left and right dACC (axial)

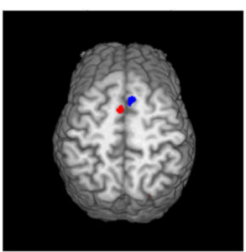

Figure 4.

Regions of brain activity differentiating repeated from corrected errors. Bars represent the mean percentage of BOLD activity for each group during repeated and corrected errors. Error bars represent the standard error of the mean. The MNI coordinates for each region are listed beside the name of each functionally defined ROI. The brain slices shown represent the view at the relevant $x, y$, or $z$-coordinate (e.g., coronal slices relate to the $y$-coordinate). The brain slice shown in $\mathrm{B} 2$ represents a composite of the left and right dorsal anterior cingulate cortices (dACC) superimposed into the same hemisphere (at the $x$-coordinate +4 ). Red represents the right $\mathrm{dACC}$ and blue represents the left $\mathrm{dACC}$ in $\mathrm{B} 2$ and $\mathrm{C} 2$. 
Table 1

Means (Standard Error) for Control $(\mathrm{n}=15)$ and Cannabis $(\mathrm{n}=15)$ Groups on Demographic and Drug Use History.

\begin{tabular}{lcc}
\hline & $\begin{array}{c}\text { Controls } \\
\boldsymbol{M}(\boldsymbol{S E})\end{array}$ & $\begin{array}{c}\text { Cannabis users } \\
\boldsymbol{M}(\boldsymbol{S E})\end{array}$ \\
\hline Age & $23.27(0.95)$ & $22.40(1.11)$ \\
Years of education & $18.27(0.76)$ & $17.13(0.52)$ \\
Verbal intelligence score (NART) & $108.51(0.38)$ & $109.02(0.77)$ \\
Beck Depression Inventory-II score ${ }^{*}$ & $2.13(0.53)$ & $5.73(1.35)$ \\
Females/males & $4 / 11$ & $2 / 13$ \\
Number of nicotine smokers & $40 \%$ & $47 \%$ \\
Years of alcohol use & $4.17(0.73)$ & $2.84(1.15)$ \\
Alcohol use in last month (no. of days) & $5.47(1.25)$ & $7.77(1.68)$ \\
Alcohol use age onset (years) & $14.39(1.68)$ & $15.60(0.46)$ \\
Cannabis use (years) & $0.00(0.00)$ & $6.43(1.07)$ \\
Lifetime joints (number) & $0.33(0.21)$ & $7341.40(2340.80)$ \\
Days of use in last month (number) & $0.00(0.00)$ & $20.80(26.66)$ \\
Joints in last month (number) & $0.00(0.00)$ & $72.47(12.60)$ \\
Cannabis use age onset (years) & & $15.97(0.42)$ \\
Cannabis abstinence (hours) & & $101.67(37.45)$ \\
Cannabis withdrawal score (out of 32) & & $12.60(2.22)$ \\
Cannabis craving scores (each item out of 21$)$ & \\
Compulsivity & & $11.13(1.10)$ \\
Emotionality & & $12.47(1.41)$ \\
Expectancy & & \\
Purposefulness & & \\
\hline
\end{tabular}

Note. NART $=$ National Adult Reading Test.

${ }^{*}<$ <.05 statistically significant difference between groups. 
Table 2

Correlations Between the Cannabis Use Composite Scores and Select Performance Indices.

\begin{tabular}{|c|c|c|}
\hline Cognitive behavioural results & Pearson's $r$ & $p$ \\
\hline Correct & -.54 & $.01^{*}$ \\
\hline Corrected error & -.28 & .23 \\
\hline Repeated error & .49 & $.02 *$ \\
\hline Error correction (\%) & -.35 & .12 \\
\hline First round recall accuracy $(\%)$ & -.55 & $.01 *$ \\
\hline Second round recall accuracy $(\%)$ & -.49 & $.02 *$ \\
\hline Recall reaction time (ms) & .50 & $.02 *$ \\
\hline Correct recall reaction time (ms) & .38 & .09 \\
\hline Error recall reaction time (ms) & .47 & $.03^{*}$ \\
\hline
\end{tabular}

* Significant at $p<.05$. 
Table 3

Regions of BOLD Activity During the Feedback Epoch (Combined Across Groups) Differentiating Corrected Errors from Repeated Errors.

\begin{tabular}{|c|c|c|c|c|}
\hline \multirow[t]{2}{*}{ Brain region } & \multirow[t]{2}{*}{ Volume $(\mu \mathrm{l})$} & \multicolumn{3}{|c|}{ MNI coordinates } \\
\hline & & $x$ & $y$ & $z$ \\
\hline \multicolumn{5}{|l|}{ Corrected errors $>$ repeated errors } \\
\hline Right putamen & 670 & 18 & 16 & 4 \\
\hline Right middle occipital gyrus & 543 & 28 & -75 & 30 \\
\hline Left hippocampus & 454 & -31 & -22 & -13 \\
\hline Right dorsal anterior cingulate cortex & 280 & 7 & 13 & 52 \\
\hline Left inferior frontal gyrus (p. opercularis) & 264 & -46 & 3 & 35 \\
\hline \multicolumn{5}{|l|}{ Repeated errors $>$ corrected errors } \\
\hline Right posterior cingulate cortex & 280 & 24 & 32 & 15 \\
\hline
\end{tabular}

Drug Alcohol Depend. Author manuscript; available in PMC 2016 October 01. 
Table 4

Regions of BOLD Activity During the Feedback Epoch Differentiating Between Controls and Cannabis Users on Combined Repeated and Corrected Errors.

\begin{tabular}{lcccc}
\hline & & \multicolumn{3}{c}{ MNI coordinates } \\
\cline { 4 - 5 } Brain region & Volume $(\boldsymbol{\mu l})$ & $\boldsymbol{x}$ & $\boldsymbol{y}$ & $\boldsymbol{z}$ \\
\hline Controls > cannabis users & & & & \\
$\quad$ Left inferior temporal gyrus & 2196 & -43 & -65 & -10 \\
Right superior parietal lobule & 1467 & 25 & -64 & 50 \\
Left inferior parietal lobule & 924 & -29 & -62 & 45 \\
Right inferior parietal lobule & 658 & 39 & -40 & 45 \\
Left hippocampus & 454 & -31 & -22 & -13 \\
Left dorsal anterior cingulate cortex & 321 & -4 & 4 & 54 \\
Right dorsal anterior cingulate cortex & 280 & 7 & 13 & 52 \\
Right thalamus & 255 & 18 & -25 & 10 \\
Left putamen & 184 & -23 & 12 & 6 \\
Left postcentral gyrus & 165 & -61 & -18 & 25 \\
Left thalamus & 147 & -9 & -20 & 11 \\
Left supramarginal gyrus & 144 & -55 & -30 & 43 \\
Right posterior anterior cingulate & 142 & 24 & 32 & 15 \\
\hline
\end{tabular}

Drug Alcohol Depend. Author manuscript; available in PMC 2016 October 01. 


\section{Table 5}

Regions of Error-Related BOLD Activity During the Feedback Epoch Indicating a Group (Controls and Cannabis Users) by Error Type (Repeated and Corrected) Interaction.

\begin{tabular}{lcccc}
\hline & & \multicolumn{3}{c}{ MNI coordinates } \\
\cline { 3 - 5 } Brain region & Volume $(\boldsymbol{\mu l})$ & $\boldsymbol{x}$ & $\boldsymbol{y}$ & $\boldsymbol{z}$ \\
\hline Left inferior temporal gyrus & 942 & -39 & -42 & 45 \\
Right inferior parietal lobule & 658 & 39 & -40 & 45 \\
Left hippocampus & 454 & -31 & -22 & -13 \\
Left dorsal anterior cingulate cortex & 321 & -4 & 4 & 54 \\
Right dorsal anterior cingulate cortex & 280 & 7 & 13 & 52 \\
Right thalamus & 255 & 18 & -25 & 10 \\
Right temporal pole & 217 & 55 & 4 & 4 \\
Left superior occipital gyrus & 212 & -22 & -86 & 37 \\
Right thalamus & 191 & 6 & -23 & 3 \\
Left putamen & 184 & -23 & 12 & 6 \\
Left thalamus & 147 & -9 & -20 & 11 \\
Left supramarginal gyrus & 144 & -55 & -30 & 43 \\
\hline
\end{tabular}

Drug Alcohol Depend. Author manuscript; available in PMC 2016 October 01. 